\title{
Measurement of the turbulent kinetic energy budget of a planar wake flow in pressure gradients
}

Xiaofeng Liu, Flint $\mathbf{0}$. Thomas

1

\section{Exp Fluids (2004) 37:469-482}

Correction 1:

In Section 3.6.2, the first line after Equation (7), the sentence "In Eq. 6, the terms $\overline{\left(\frac{\partial u_{1}^{\prime}}{\partial x_{1}}\right)^{2}}$ and $\overline{\left(\frac{\partial u_{1}^{\prime}}{\partial x_{3}}\right)^{2}}$..." should be read as "In Eq. 6, the terms $\overline{\left(\frac{\partial u_{1}^{\prime}}{\partial x_{1}}\right)^{2}}$ and $\overline{\left(\frac{\partial u_{2}^{\prime}}{\partial x_{1}}\right)^{2}}$..."

\section{Correction 2:}

In Section 4.1, the second sentence : “... and the local maximum velocity defect $U_{\mathrm{d}}(\mathrm{y})$ as the reference velocity scale" should be read as "... and the local maximum velocity defect $U_{d}(x)$ as the reference velocity scale." Correction 3:

In Section 4.3, the first sentence "... the streamwise turbulence diffusion $-\frac{\partial}{\partial x_{1}} \overline{\frac{1}{2}\left(u_{1}^{\prime 3}+u_{1}^{\prime} u_{1}^{\prime 2}+u_{1}^{\prime} u_{3}^{\prime 2}\right)}$ and ..." should be read as "... the streamwise turbulence diffusion $-\frac{\partial}{\partial x_{1}} \overline{\frac{1}{2}\left(u_{1}^{\prime 3}+u_{1}^{\prime} u_{2}^{\prime 2}+u_{1}^{\prime} u_{3}^{\prime 2}\right)}$ and ...”

E-mail: Flint.O.Thomas.1@nd.edu 